\title{
Aesthetic Considerations in Forehead Reconstruction in Skin Carcinoma
}

\author{
De Abullarade, Jezabel \\ Plastic and Reconstructive Surgery Department of the Instituto Salvadoreño del Seguro Social, San Salvador, El Salvador, Central \\ America. \\ Email: drdeabullarade@yahoo.com
}

Received May 23 ${ }^{\text {rd }}, 2013$; revised June $22^{\text {nd }}, 2013$; accepted June $30^{\text {th }}, 2013$

Copyright (C) 2013 De Abullarade, Jezabel. This is an open access article distributed under the Creative Commons Attribution License, which permits unrestricted use, distribution, and reproduction in any medium, provided the original work is properly cited.

\begin{abstract}
Reconstruction of the forehead has been a challenge for many surgeons. Some use skin grafts, other microvascular flaps, but local flaps for coverage of forehead skin are still the cornerstone principle in providing the ideal skin color and texture. In this article, the forehead is classified into surgical reconstructive subunits. These subunits are set according to the surrounding structures. Flaps are rectangular in shape in mobility in order to follow the natural lines of the forehead.
\end{abstract}

Keywords: Forehead; Flap; Rectangle; Areas; Zone

\section{Introduction}

Eighty six percent of basal cell carcinomas are found on the head, and $12.5 \%$ of these are located on the forehead [1], the forehead as one unit that comprises a third of the face. Therefore it is more difficult to hide it from the human eye. This is the challenge for a reconstructive surgeon.

The surgeon needs to resurface the same characteristics of color, texture, thickness of the forehead. Obtaining these characteristic by advancing local flaps in order to resurface a skin defect in this area is difficult. It is even more complex due to the inelasticity tissue and the neighboring hairline or eyebrows.

\section{Reconstructive Focus of Skin Cancer}

Most patients wish the missing part of the forehead restored to its original characteristics and contour, although they are not always vocal about this. Patients are sensitive about their appearance. They do not as concern about which technique the surgeon is going to use, they only want to look as they did before [2] and this is the challenge for a reconstructive surgeon.

No one in the world can turn back the original structures; however, we can somehow camouflage scars, creating an illusion to the eye of the observer. We can hide the scars in the wrinkles and the edges of the forehead.

The relationship with the surrounding structures of the wound is critical. Anatomically the forehead is surround- ed by the hairline distally and eyebrows proximally, which are structures that cannot be moved in order to pursue optimal results. Therefore the contour plane or surface must not be change.

The forehead is comparable anatomically to a nearly concave rectangle as a single unit. To camouflage scars we have to imitate this rectangle, in small to moderate defects.

The use of skin graft as well as microsurgical flaps is not an optimal choice due to the incompatibility of color and texture [3] and thickness. Even using local flaps, if they are not based in the geometrical orientation of the forehead allowed the scars to remain visible to the observer.

To achieve superior results in facial reconstruction, and in this particular issue, the reconstruction of the forehead, we designed sub units named "surgical zones". These Zones follows the natural wrinkles and geometric contours of the forehead. Two kinds of rectangular flaps have been designed: "the sliding curtain flap" and "sliding door flap" as described later.

By drawing imaginary lines according to the adjacent structures in each area we designed sub units and named them: Zone I, Zone II, Zone III and Zone IV (Figure 1).

In order to obtain the most natural reconstruction of the forehead, three parallel horizontal lines were designed. The first line is located on the edge of the hairline, the second line is on the halfway point of the forehead, and third in the top of both eyebrows. The lateral borders 


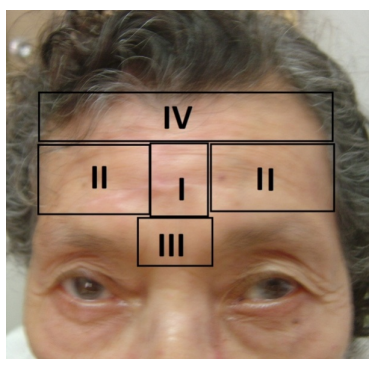

Figure 1. Forehead unit classified on four surgical zones.

of the forehead are both edges of the temporal areas and in the middle of the forehead there are two parallel vertical lines starting at each medial edge of the eyebrows.

\section{Material and Methods}

Forty six patients have been operated by the senior author in a period of 10 years, from 2002 to 2012. These patients underwent forehead reconstruction after ablation with tumor free margins of skin cancer at the Hospital Roma of the Instituto Salvadoreño del Seguro Social.

These patients were refered by the Dermatology Department. They were diagnosed with primary lesions of basal cell cancer in forehead. One patient had the diagnosis of squamous cell cancer.

The patients were $56 \%$ male and $44 \%$ female with range age of 28 to 95 years old.

Surgical reconstruction zones were based on the geometry of the forehead, the rhytides and surrounding structures, such as the hairline distally, proximally and laterally eyebrows temporal regions, as we described before (Figure 1).

\subsection{Zone I}

Zone I refers to skin defects in the central area of the forehead, where no surrounded structures exist.

The defect in this zone is very difficult to hide because it is often the first part of the forehead that people observed.

Zone I is designed as a rectangle drawn into two parallel lines that start at each medial edge of the eyebrows, and whose base is a straight horizontal line that goes at the top of both eyebrows and the upper edge is a straight horizontal line that is drawn in the middle of the forehead.

In this Zone I is designed double sliding door flaps, which are rectangular cutaneous-sub galeal flaps one on the other side of the defect, following natural wrinkles, the flaps are elevated and advanced to the center of the defect (Figure 2).

\subsection{Zone II}

Zone II is the two areas over the eyebrows, one area over

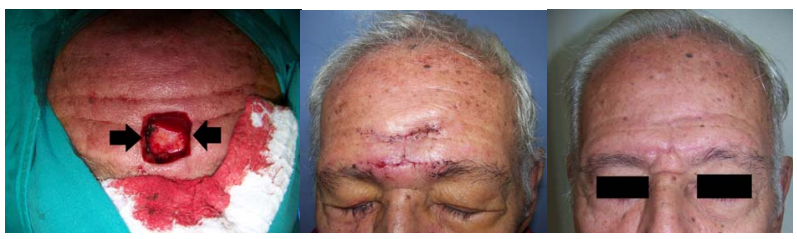

Figure 2. Demonstrating the post resection of skin basal cell cancer defect in Zone I. Bilateral sliding door flaps. Six months post operatory result.

the right and one area over the left include all area over each eyebrow. The proximal border of each area in this Zone is a horizontal line over each eyebrow, and the distal border is a horizontal line drawn at the middle of the forehead, following the wrinkles, limited laterally by the medial border of each temporal areas.

The stronger challenge that is faced in Zone II is to maintain the integrity of the eyebrows.

In order to consider this issue, there are three main kinds of defects that the surgeon faces:

When the defect is medial in Zone II and moderate size we designed a simple sliding door flap, drawing a rectangular flap temporal based, elevated and advanced to the center of the defect (Figure 3).

If the defect is smaller in Zone II and medial, a sliding curtain flap is designed.

This flap is elevated subgaleal and advanced to the medial border of the defect of Zone II over the upper edge of the eyebrow. The difference in this flap from the sliding door flap is, that this sliding curtain flap is elevated and advanced by one border, distal or proximal border (Figure 4).

When the defect in Zone II is located at the distal border of the eyebrow in the forehead, in the middle of zone II, a double sliding door flap should be advanced (Figure 5).

\subsection{Zone III}

This Zone III is the glabellar area, which is very complex due to the anatomical area between the eyebrows. The challenge is to maintain the medial edge eyebrow border and the glabellar area integrity.

In Zone III a double sliding curtain flap was design, as described before, with the inferior border of the flap following the distal margin of each eyebrow, and elevating the flap subgaleal and advancing it to the center of the defect. At the same time that a radix nasal flap is designed and advanced to the remaining defect if necessary. The lateral borders of this radix cutaneous flap are the medial canthal borders, and is elevated submuscular to the remaining defect (Figure 6).

\subsection{Zone IV}

Zone IV is located at the proximity of the hairline. This 


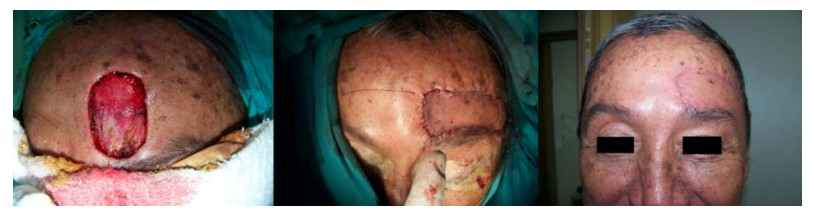

Figure 3. Patient with the defect in Zone II. Reconstructed defect with a simple siding door flap with temporal based. Three months after reconstruction.

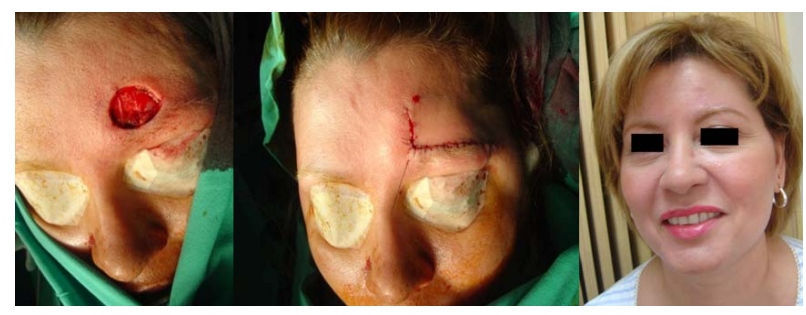

Figure 4. Patient with a smaller defect over the medial side of the eyebrow in Zone II. Immediate reconstruction with a sliding curtain flap. Full face of the patient after six months.

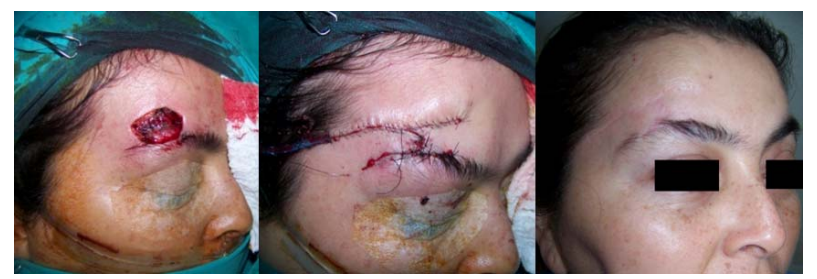

Figure 5. Post resection defect in Zone II. Immediate reconstruction with double sliding door flap. Same patient after six months of reconstruction.

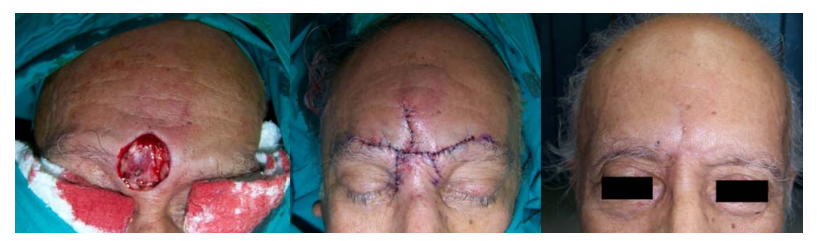

Figure 6. Defect in Zone III. Immediate reconstruction with double sliding curtain flap and radix flap. Six months result.

Zone IV is limited distally by the hairline and proximally by a horizontal line drawn in the middle of the forehead; the laterals borders of this zone are the medial edges of each temporal areas.

Maintaining the integrity of the hairline is the goal of the reconstruction of the defects in Zone IV. To achieve this goal, a double curtain flap with hairline as it based is design in this Zone (Figures 7 and 8).

To achieve reconstructions with complex defects as those that encompass two zones, it is necessary to overlap flaps and add relaxing galeal incisions (Figure 9).

\section{Results}

All patients underwent the classification of the sub units

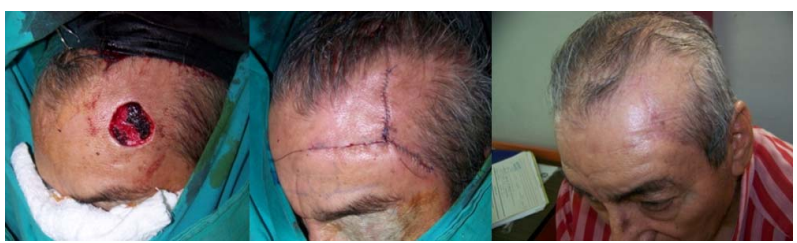

Figure 7. Defect in Zone IV after resection of basal cell carcinoma. Immediate reconstruction. Result after six months.

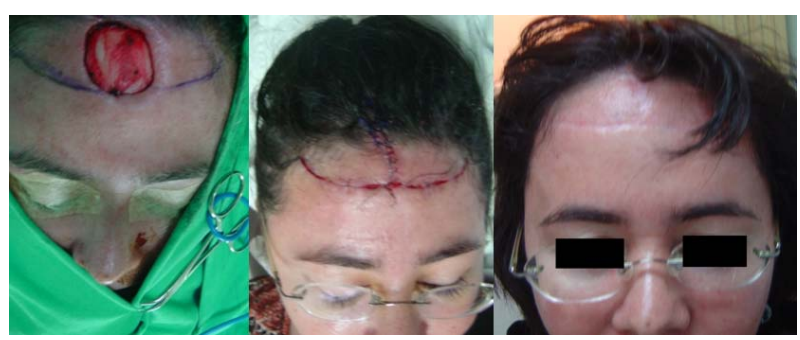

Figure 8. Defect in zone IV after ablation of basal cell carcinoma. Reconstruction with bilateral curtain fap. Result after six months.

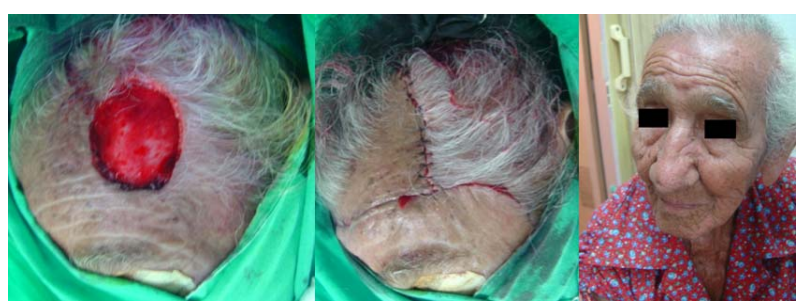

Figure 9. Complex defect due to the resection of basal cell carcinoma in Zone II and IV. Reconstruction with double curtain flap. Result after six months.

by surgical Zones.

The reconstruction was performed using sliding door flaps, single or double flap or by using curtain flaps.

There was no partial or total flap loss, no infections. The face edema resolved gradually one week after the procedure.

The aesthetic result was excellent in all patients after six months to a year of follow up.

A patient with a complex defect which resulted a hypertrophic scar, was resolved after a revision.

All patients and family were pleased with the results.

\section{Discussion}

There are several techniques of reconstruction of the forehead written in the literature, however, the forehead unit remains a challenge for all reconstructive surgeons.

It is preferable to reconstruct with adjacent tissue as much as possible, due to the stronger results obtained with the same kind of tissue, and the use of the sub unit principle in the forehead. This also led to the use of a new surgical classification and the design of the sliding door and sliding curtain flaps. These solutions were eas- 
ily performed in the reconstruction process as was shown in the patients' profiles. Although local flaps have limitations regarding the size of defects that can be closed, these limitations can be easily overcome by applying this Zone classification and newly designed flaps.

The different reconstructive techniques that consider the size of the defect on the forehead are well known. The simplest direct closure is limited to defects less than an inch, due to the type of inelastic tissue in which we are working.

The skin graft [3] would appear as a misplaced patch on the forehead. Regardless of the color and texture of the skin graft, they are unpredictable and frequently become pigmented, shiny or atrophic, unless the skin graft is used to reconstruct the whole unit.

Some authors prefer a varied number of reconstructions such as myocutaneous [4] and others use expansion [5] or even microsurgery reconstructive methods like radial forearm flap, latissimus dorsi flap, each with its advantages and disadvantages to consider as well as to consider the size of the defect. The cutaneous island flaps [6] or myocutaneous flaps, can leave bulky deficiency and different skin quality characteristics as well as unpredictable scar position at the donor site. These methods provide no advantages over the use of local flaps.

It have been demonstrated that the use of the sub unit classification in four different surgical Zones, orienting the design of the local flaps according to the surrounded structures is a superior aesthetic solution.

\section{Conclusions}

This article presents the development of a simple classification based on the sub unit principle and designed local flaps that are practical and aesthetic, resolving great number of post skin cancer surgery defects. The classification and the local flaps designed were effective across ages and varieties of defects as demonstrated in the included photographs.

By adding this surgical classification, it has been possible to achieve excellent aesthetic results in one single stage without disrupting fixed structures such as the hair- line or the eyebrows.

Excellent reconstructive results with simple as well as complex defects have been achieved. The more complex defects are those that encompass two zones in which it is necessary to overlap flaps along with relaxing galeal incisions.

The classification of Zones and the design of local sliding door and curtain flaps are additional tools for the plastic surgeon to use in the reconstruction of the forehead.

\section{Acknowledgements}

In loving memory of my husband Dr. Jose Eduardo Abullarade Dahbura, August 1956-December 2012.

The author offers many thanks to Susan Heckmann for her English editing assistance.

\section{REFERENCES}

[1] N. David and S. Melvin, "Basal Cell Carcinoma: An Overview of Tumor Biology and Treatment,” Plastic \& Reconstructive Surgery, Vol. 113, No. 5, 2004, p. 75e.

[2] J. De Abullarade, "Versatility of Cheek Zigomatic Flap in Nose Reconstruction,” Cirugía Plástica Ibero Latinoamericana, Vol. 38, No. 3, 2012, p. 274.

[3] R. Gurunlouglu, et al., "Reconstruction of Large SupraEyebrow and Forehead Defects Using Hatchet Flap Principle and Sparing Sensory Nerve Branches,” Annals of Plastic Surgery, Vol. 68, No. 1, 2012, pp. 37-42. doi:10.1097/SAP.0b013e3182110fce

[4] A. Gevorgyan, et al., "Supraorbital Artery Myocutaneous Island Flap for Forehead Defect Reconstruction,” Journal of Craniofacial Surgery, Vol. 19, No. 2, 2008, pp. 513516. doi:10.1097/SCS.0b013e31806900fa

[5] A. K. Gosain, et al., "Refinements of Tissue Expansion in Pediatric Forehead Reconstruction: A 13 Year of Experience,” Plastic \& Reconstructive Surgery, Vol. 124, No. 5, 2009, pp. 1559-1570.

[6] J. Guerrerosantos, "Frontalis Musculocutaneous Island Flap for Coverage of Forehead Defects," Plastic \& Reconstructive Surgery, Vol. 105, No. 1, 2000, p. 18. doi:10.1097/00006534-200001000-00003 